\title{
Etude des cernes d'accroissement ligneux du système racinaire d'arbres traités en taillis
}

\author{
M. BEDENEAU et L. PAGES \\ I.N.R.A., Station de Recherches sur la Foret et l'Envirounement \\ Ardon, F 45160 Olivet
}

\section{Résumé}

Afin de déterminer le mode de renouvellement des grosses racines d'arbres forestiers après recépage, des souches de deux essences (bouleau et châtaignier), traitées en taillis, ont été extraites. Des rondelles ont été prélevées à la partie proximale des plus gros éléments du système racinaire.

Les cernes de ces rondelles ont été dénombrés par moyens optiques ou par radiographies. Le caractère annuel de la croissance radiale des racines a été mis en évidence notamment par la mesure au ${ }^{31} \mathrm{C}$ d'origine thermonucléaire.

Les résultats, obtenus à partir de l'examen de trois souches de bouleau et de sept souches de châtaignier, montrent que l'âge des racines de bouleau est égal à l'âge total de la souche, en revanche l'âge des racines de châtaignier est inférieur à celui de la souche.

\section{Introduction}

Nous voudrions d'abord insister sur quelques points importants :

- cette étude a été réalisée dans la Région Centre (France), sur 4 parcelles;

- la lourdeur de l'expérimentation, difficile à éviter dans ce genre d'étude (voir photo 1), n'a permis d'extraire que 10 souches;

- la présomption de la périodicité annuelle des cernes racinaires est forte, mais des travaux restent encore à faire pour la démontrer parfaitement.

En tenant compte de ces réserves, qui veulent simplement éviter une généralisation fâcheuse, on peut faire les remarques suivantes :

Il est bien connu que sous les climats tempérés, les arbres se développent au printemps et à l'été et cessent leur croissance en automne et en hiver. Cette discontinuité de fonctionnement est à lorigine des cernes annuels, visibles sur la coupe radiale du tronc d'un arbre. Lorsque les cernes sont dénombrés, on obtient l'âge de l'arbre.

Traditionnelle sur les parties aériennes (tronc et branches), cette méthode est peu utilisée pour les parties souterraines de l'arbre (racines). Ceci est dû au fait que la périodicité de croissance radiale des parties souterraines n'est pas absolument prouvée. 
De nombreux auteurs considèrent la croissance racinaire comme annuelle (LAITAKAR1, 1935 ; STOUT, 1956 ; FAYLE, 1969 ; FRITTS, 1976). Le renouvellement des racines après recépage varie selon les espèces, utilisation de l'ancien système racinaire chez l'eucalyptus (RIEDACKER, 1973), formation de nouvelles racines chez l'érable (LEEs, 1981). RiedACKer considère qu"il y a arrêt de croissance radiale des racines lors du recépage.

C'est pourquoi nous avons utilisé des méthodes complémentaires de datation, afin de dénombrer les cernes des parties souterraines de souches d'arbres traités en taillis, et en démontrer le fonctionnement périodique.

Cette étude s'inscrit dans le cadre de recherches sur laccroissement de la production de biomasse ligneuse, notamment à des fins énergétiques. Il est done important de comprendre la croissance des parties souterraines des taillis afin d'en maximiser le rendement des parties aériennes.

\section{Matériel et méthode}

L'étude s’est déroulée dans la Région Centre (France). Dans cette région, le taillis représente 67 p. 100 de la surface boisée, le taux de boisement étant de 38 p. 100 (environ 25 p. 100 de la surface totale est donc boisée en taillis).

Deux essences ont été retenues:

- le bouleau : espèce pionnière, partout présente dont la production en taillis est intéressante (AUClair \& METAYER, 1980);

- le châtaignier : également présente partout sous le régime du taillis.

Les souches portant des rejets ont été choisies dans des parcelles dont lhistoire est connue grâce à des archives spécifiques (tabl. 1). Du fait de la différence morphologique du système racinaire de ces deux essences (traçant pour le bouleau, plutôt pivotant pour le châtaignier), des techniques différentes ont été utilisées pour l'extraction.

\subsection{Bouleau}

\subsection{Extraction}

Selon une méthode de BoHm (1979), une tranchée circulaire a été creusée à 2 mètres autour de l'arbre retenu. Le bloc cylindrique ainsi défini a été aspergé avec de l'eau sous pression, dans le but d'ôter la terre englobant les racines. L'évacuation de la terre se fait sur place (photo 1), il reste alors l'ensemble du système racinaire de diamètre supérieur à $1 \mathrm{~cm}$, qui est transporté au laboratoire pour analyse. Trois souches ont été extraites (PAgEs, 1982).

\subsection{Châtaignier}

Nous avons utilisé la technique décrite par LeEs (1981). L'extraction des souches est faite à l'aide d'un «tracto-pelle», qui commence à creuser une tranchée circulaire de 1,5 mètre de profondeur à une distance de 2 mètres de la cépée. Les grosses racines sont coupées au godet, après un tour complet de l'engin, la souche n'a plus qu’à être soulevée, puis chargée. 
TABIEAU 1

Echantillon do souches de taillis.

Sampling of coppice stumps.

\begin{tabular}{|c|c|c|c|c|}
\hline & Essence & Age parties aériennes & Nombre de rotations & Durée de la rotation \\
\hline \multicolumn{5}{|c|}{ Bouleau } \\
\hline 1 & & 9 & 1 & 25 \\
\hline 2 & $\cdots$ & 14 & 1 & 25 \\
\hline 3 & $\ldots$ & 24 & 1 & 25 \\
\hline \multicolumn{5}{|c|}{ Châlaignier } \\
\hline 1 & $\ldots$ & 18 & 1 & 20 \\
\hline 2 & $\ldots$ & 14 & 1 & 15 \\
\hline 3 & $\ldots \ldots \ldots$ & 19 & 1 & 20 \\
\hline 4 & $\ldots$ & 14 & 1 & 15 \\
\hline 5 & & 13 & 1 & 15 \\
\hline 6 & $\ldots \ldots$ & 16 & 1 & 15 \\
\hline 7 & & 35 & 2 & 35 \\
\hline
\end{tabular}

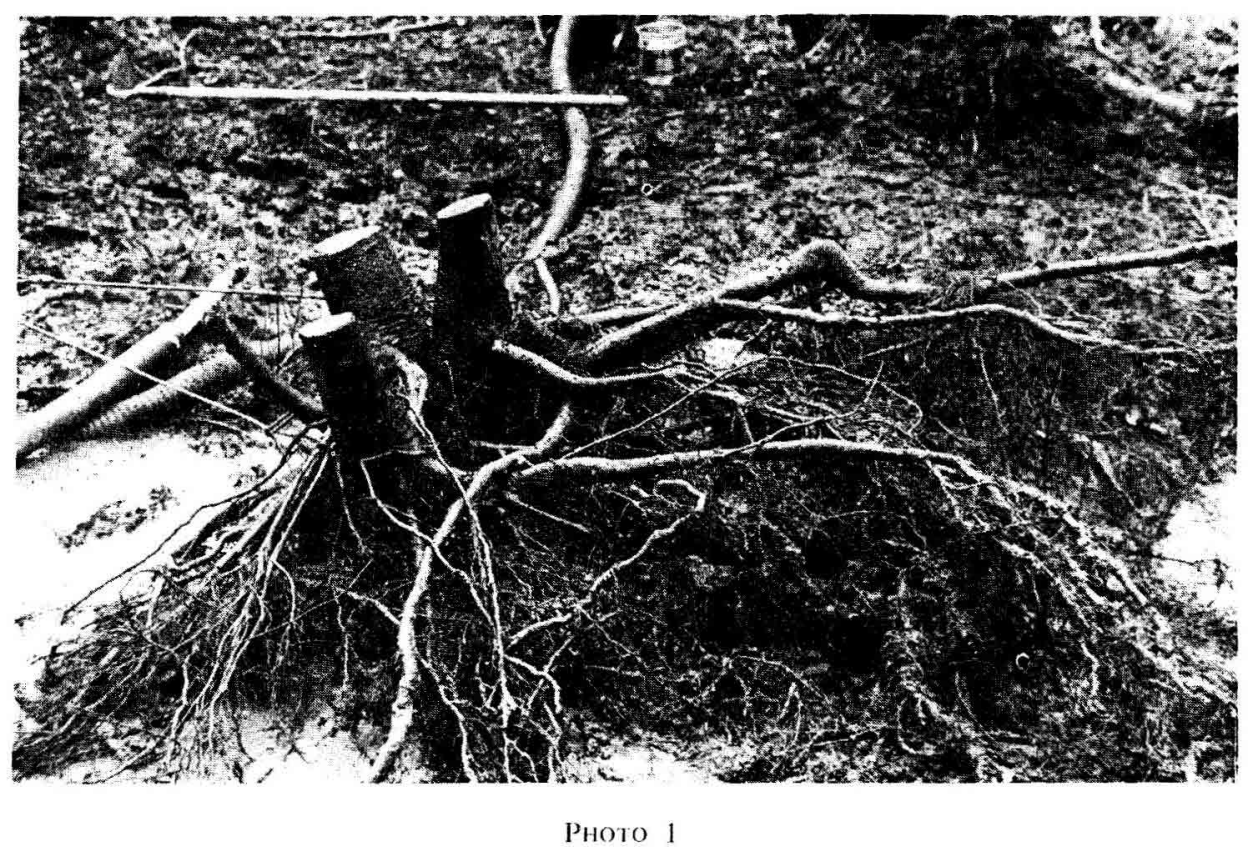

Extraction d'une souche de bouleau.

Extraction of a birch stump. 
A la différence du bouleau, la terre est enlevée au jet après transport. Pour des souches plus petites, l'extraction se fait au treuil. Sept souches ont été prélevées (photo 2).

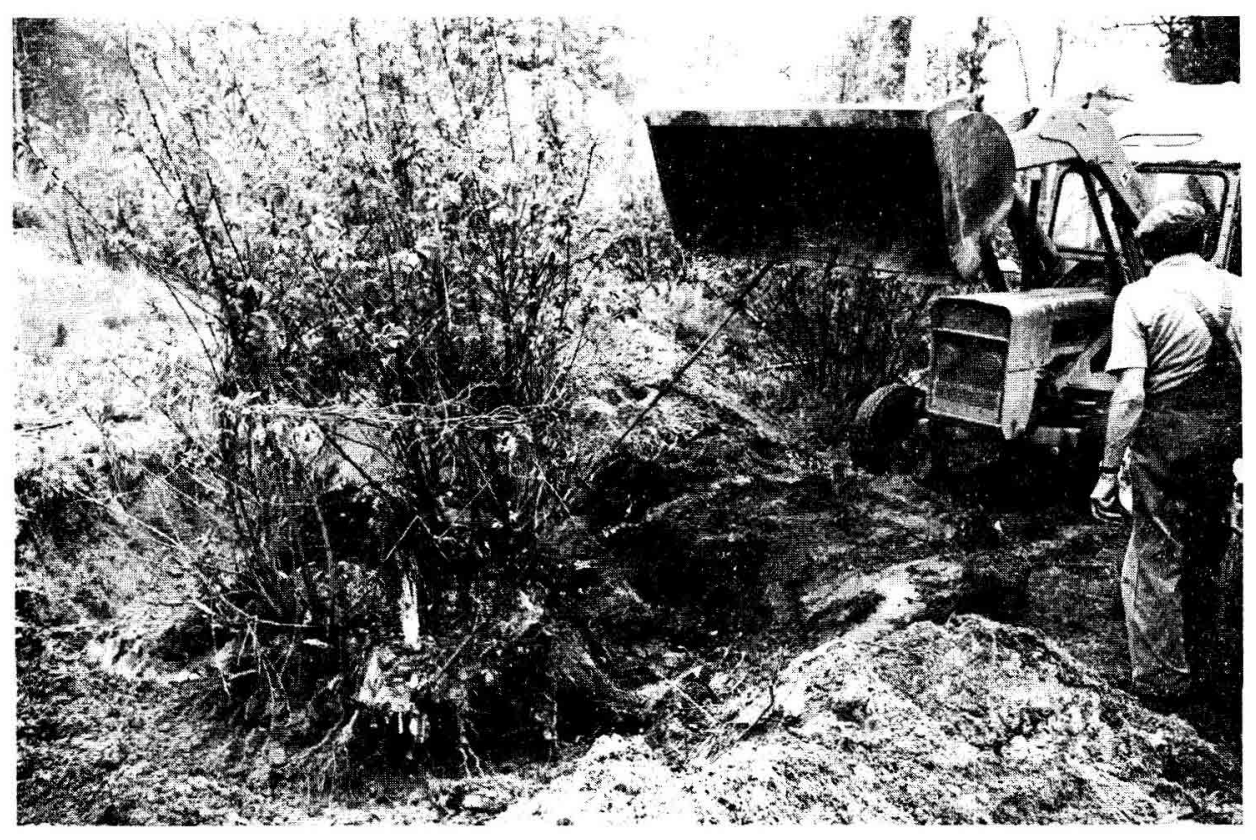

PHOTO 2

Extraction diune souche de châtaignier.

Extraction of a chesnut stamp.

\subsection{Echantillonnage}

Des considérations pratiques (PAGES, 1982) nous ont conduit à ne retenir que les racines de diamètre proximal supéricur à $5 \mathrm{~cm}$. Parmi ces racines, on a choisi les plus grosses, qui ont été repérées et numérotées (1). Une rondelle de bois est prélevée à la partic proximale de ces racincs, puis soigneusement poncée.

\subsection{Lecture}

Chaque rondelle a été placéc sous loupe binoculaire et les cernes dénombrés par 4 observateurs différents, sur 2 rayons. Chez le bouleau, les cernes racinaires sont très fins et très peu discernables par cette méthode. Les rondelles les plus illisibles ont été radiographiécs selon la technique de BedenEaU \& Rimsky (1976).

(1) Selon cette méthode, Il racines ont d'abord été choisies sur la souche de catégorié n" 7 . Puis, vu l'intérêt de cette souche. l'ensemble des racines de $y=5 \mathrm{~cm}$ a été retenu. 
Les films résultants sont placés sur une table lumineuse, sous loupc binoculaire. La lecture est alors possible.

Dans tous les cas, la datation obtenue est la moyenne des quatre observations, avec des écarts maximaux de \pm 2 ans.

\subsection{Datation}

L'âge des souches étant connu (voir supra 1), il sagit de connaître la périodicité des cernes racinaires.

Pour le bouleau, le caractère annuel de l'accroissement radial a été mis en évidence à l'aide $\mathrm{du}{ }^{14} \mathrm{C}$ d'origine thermonucléaire. Cette méthode consiste à doser le ${ }^{14} \mathrm{C}$ contenu dans les cernes, et à comparer le résultat de ce dosage à des valeurs de la courbe d'évolution du ${ }^{1}{ }^{4} \mathrm{C}$ dans l'atmosphère. La précision obtenue est de \pm 2 ans (Pages, 1982 ; Pages et al., sous presse).

De telles analyses noont pu être réalisées sur le châtaignier, c'est pourquoi on a utilisé plusieurs méthodes complémentaires :

- à l'aide de carnets-affiches et de plans de balivages, l'histoire de la parcelle d'où ont été extraites les souches (2) a été reconstituée depuis 1885 ;

- les plus vieilles souches ont été fendues et les cernes de la base des brins suivis jusque dans les racines;

- les «chicots» des rotations précédentes ont été datés par dénombrement et recoupement avec les carnets-affiches, les cernes aériens suivis jusque dans la partie souterraine.

La confrontation des différentes méthodes utilisées pour dater les cernes racinaires du châtaignier nous donne la présomption que les cernes sont annuels.

\section{Résultats et discussion}

Les résultats sont de deux types :

- qualitatifs : observation sur la forme des racines, la disposition des cernes;

- quantitatifs : nombre de cernes racinaires, regroupés dans le tableau 3.

Une différence notable apparaît à l'examen des rondelles de racines. Les racines de châtaignier sont toutes plutôt circulaires, qu'elles soient latérales ou pivotantes.

Les racines latérales de bouleau ont un aspect ovale qui est dû à une croissance d'abord bien répartie autour du cœur, puis devenant par la suite plus forte dans les parties inférieures et supérieures (photo 3).

Les racines pivotantes ont un contour plutôt triangulaire.

(2) Forêt domaniale de Lamotte-Beuvron (Loir-et-Cher). 

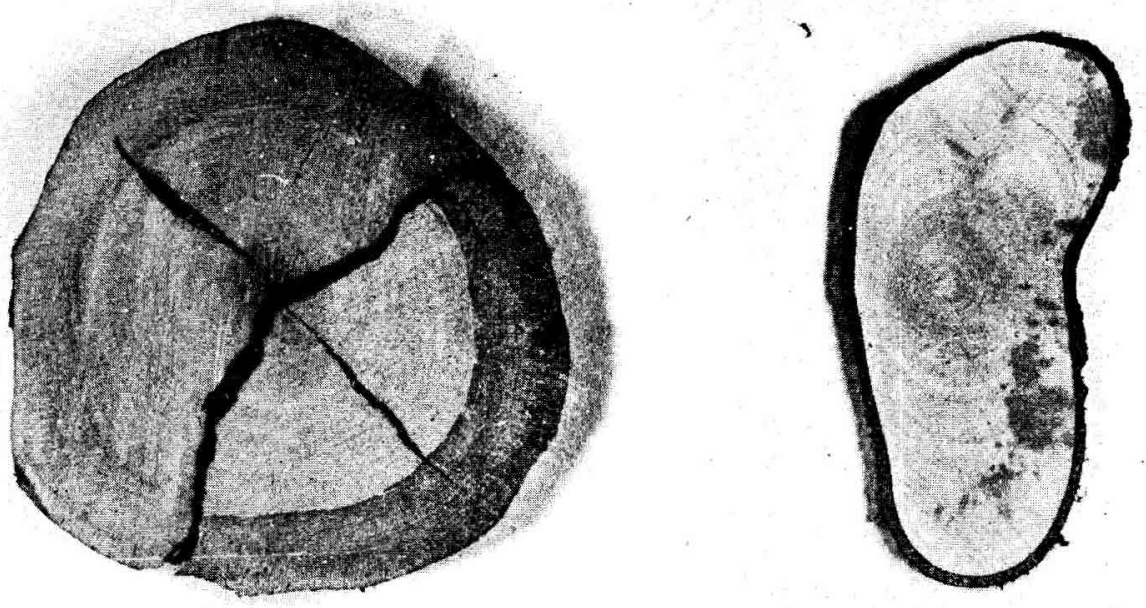

Рното 3

Différence de forme entre racine de bouleau et de châtaignier (voir texte).

Difference between the form of birch root and chesnut root (see text).

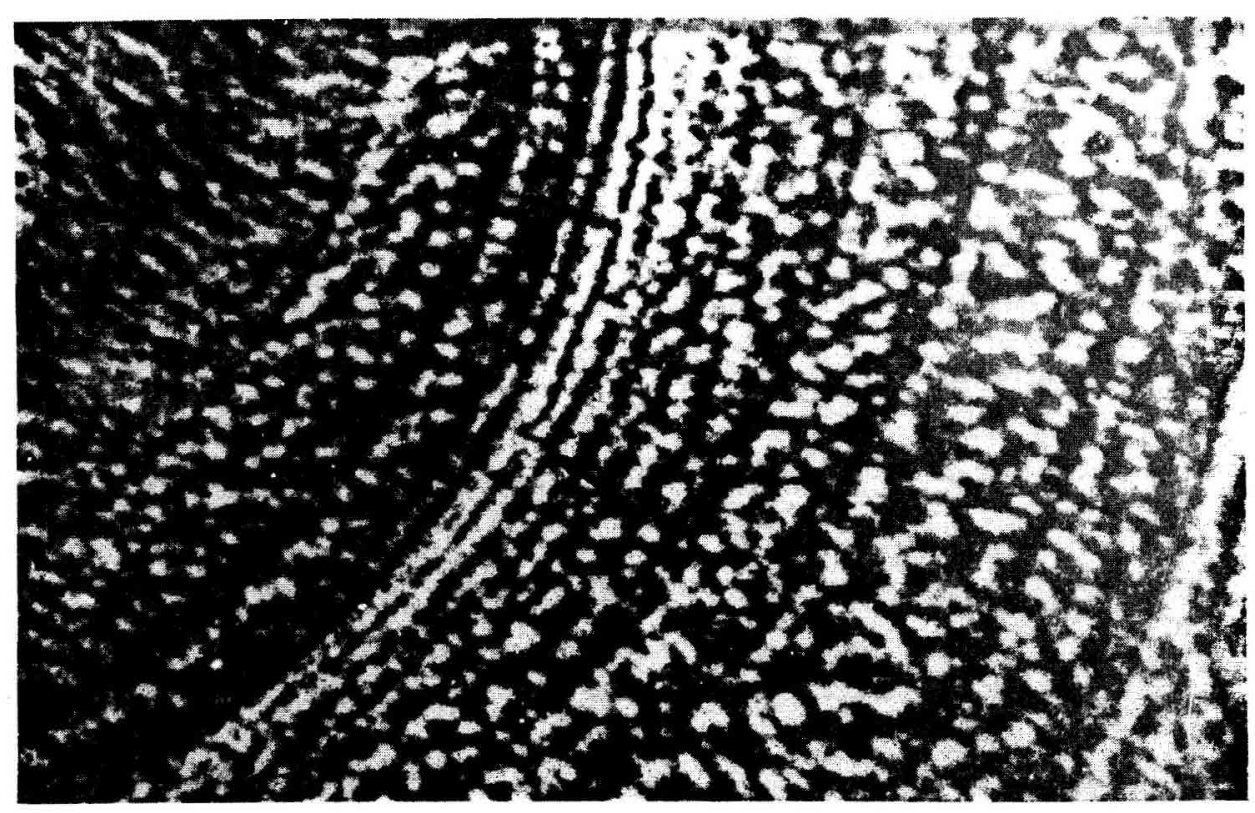

\section{Рното 4}

Reserrement des cernes racinaires de bouleau à l'époque du recépage. Narrow root rings of birch, following the period of cutting. 
TABLEAU 2

Echantillonnage des racines.

Sampling of roots.

\begin{tabular}{|c|c|c|c|}
\hline Essence & $\mathrm{N}^{\circ}$ & $\begin{array}{c}\text { Nombre de racines } \\
\text { de la souche } \\
\text { de diamètre } \geqslant 5 \mathrm{~cm}\end{array}$ & $\begin{array}{c}\text { Nombre de racines } \\
\text { étudiées }\end{array}$ \\
\hline Bouleau & $\begin{array}{l}1 \\
2 \\
3\end{array}$ & $\begin{array}{l}7 \\
6 \\
7\end{array}$ & $\begin{array}{l}2 \\
3 \\
4\end{array}$ \\
\hline Châtaignier & $\begin{array}{l}1 \\
2 \\
3 \\
4 \\
5 \\
6 \\
7\end{array}$ & $\begin{array}{r}5 \\
6 \\
4 \\
4 \\
5 \\
6 \\
33\end{array}$ & $\begin{array}{r}2 \\
2 \\
2 \\
4 \\
5 \\
4 \\
11(+22)\end{array}$ \\
\hline
\end{tabular}

Au comptage, on observe sur les racines de bouleau (pivotantes ou traçantes) une série de 2 à 4 cernes très étroits (photo 4 ); la racine avait alors 25 ans, la partie aérienne venait d’être coupée.

Aucune séquence similaire n'est vis:ble sur les racines de châtaignier.

Sur le tableau 3, il apparaît que lâge des racines de bouleau est égal, à un cas près (racine 4 du bouleau 3) à l'âge total de la souche.

La représentation graphique du tableau 3 (fig. 1) montre que les racines de châtaignier sont situées dans tous les cas sous la première bissectrice (âge des racines inférieur à l'âge total de la souche). Les racines de bouleau, dans 8 cas sur 9, sont placées sur cette première bissectrice (égalité entre âge des racines ct âge total de la souche).

On peut donc penser qu'après recépage le bouleau «repart » sur l'ancien système racinaire, alors que le châtaignier réémet à chaque rotation de nouvelles racines. Pour le bouleau, il n'y a pas d'extension du système racinaire primaire et peu de renouvellement des racines charpentières. La forme ovale des racines latérales pourrait alors être due aux compressions exercées par les parties aériennes (FAYl.L, 1968). Le rétrécissement des cernes, correspondant à l'époque du recépage, est une preuve supplémentaire de la réutilisation du système racinaire par les nouveaux rejets.

En revanche, le châtaignier renouvelle perpétuellement ses racines : sur la souche de 105 ans, les écarts d'âges entre souche et racines vont jusqu'à 80 ans, et pour les souches de 28-29 ans, ces écarts atteignent 15 à 20 ans. 
Tableau 3

Résultats des comptages.

Results of rings counts.

\begin{tabular}{|c|c|c|c|c|}
\hline Essence & $\mathrm{N}^{\prime \prime}$ racine & $\begin{array}{l}\text { Nombre } \\
\text { de cernes } \\
\text { racinaires }\end{array}$ & $\begin{array}{c}\text { Age } \\
\text { partie aérienne }\end{array}$ & Age souche \\
\hline Bouleau 1 & $\begin{array}{l}1 \\
2\end{array}$ & $\begin{array}{l}34 \\
34\end{array}$ & 9 & 34 \\
\hline Bouleau 2 & $\begin{array}{l}1 \\
2 \\
3\end{array}$ & $\begin{array}{l}39 \\
39 \\
39\end{array}$ & 14 & 39 \\
\hline Bouleau 3 & $\begin{array}{l}1 \\
2 \\
3 \\
4\end{array}$ & $\begin{array}{l}49 \\
47 \\
47 \\
20\end{array}$ & 24 & 49 \\
\hline Châtaignier 1 & $\begin{array}{l}1 \\
2\end{array}$ & $\begin{array}{l}18 \\
19\end{array}$ & 18 & 38 \\
\hline Châtaignier 2 & $\begin{array}{l}1 \\
2\end{array}$ & $\begin{array}{l}17 \\
12\end{array}$ & 14 & 29 \\
\hline Châtaignier 3 & $\begin{array}{l}1 \\
2\end{array}$ & $\begin{array}{l}10 \\
22\end{array}$ & 19 & 39 \\
\hline Châtaignier 4 & $\begin{array}{l}1 \\
2 \\
3 \\
4\end{array}$ & $\begin{array}{l}13 \\
15 \\
17 \\
14\end{array}$ & 14 & 29 \\
\hline Châtaignier 5 & $\begin{array}{l}1 \\
2 \\
3 \\
4 \\
5 \\
\end{array}$ & $\begin{array}{r}10 \\
12 \\
8 \\
13 \\
13 \\
\end{array}$ & 13 & 28 \\
\hline Châtaignier 6 & $\begin{array}{l}1 \\
2 \\
3 \\
4\end{array}$ & $\begin{array}{l}16 \\
15 \\
12 \\
18\end{array}$ & 16 & 31 \\
\hline \multirow[t]{2}{*}{ Châtaignier 7} & $\begin{array}{r}1 \\
2 \\
3 \\
4 \\
5 \\
6 \\
7 \\
8 \\
9 \\
10 \\
11\end{array}$ & $\begin{array}{l}40 \\
38 \\
38 \\
39 \\
57 \\
36 \\
38 \\
57 \\
84 \\
44 \\
26\end{array}$ & 35 & 105 \\
\hline & $\begin{array}{c}12 \\
13-14 \\
15 \\
16-33\end{array}$ & $\begin{array}{l}37 \\
32 \\
33 \\
<30\end{array}$ & & \\
\hline
\end{tabular}




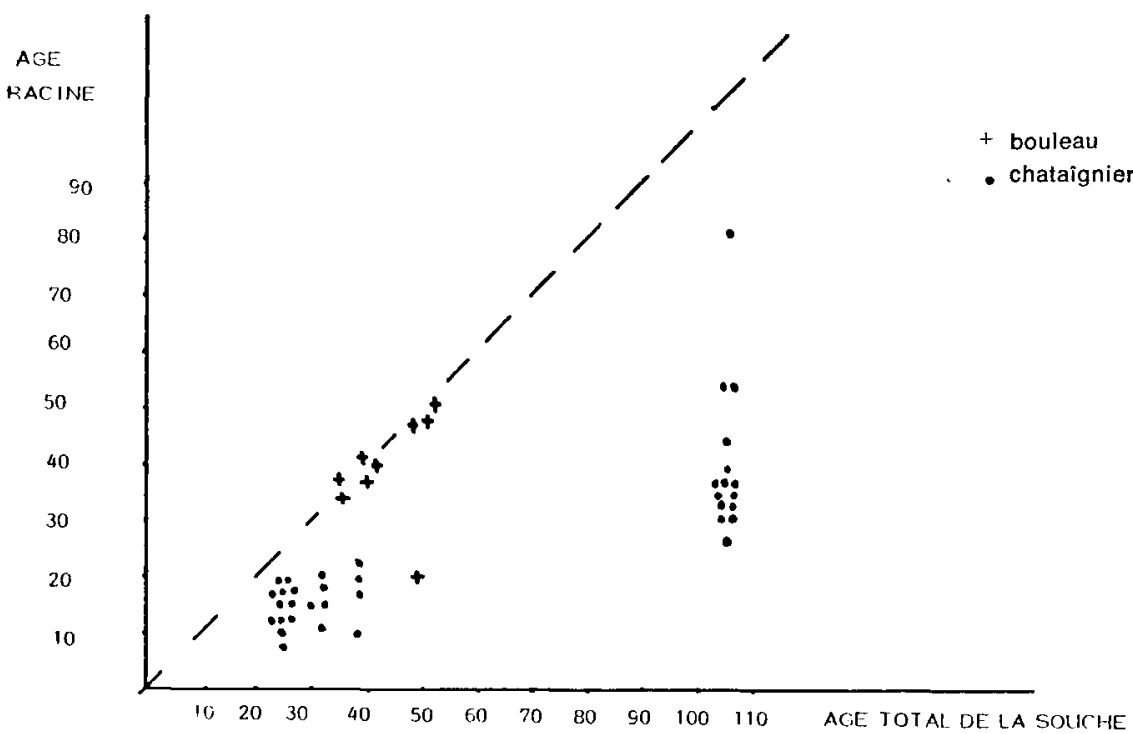

FIG. 1

Représentation graphique des résultats de comptage.

Graphical representation of rings counts.

On peut comprendre que les souches de bouleau s'épuisent plus vite que les souches de châtaignier, du fait que l'ensemble du système racinaire reste plus longtemps en place (sénescence, attaque de la flore microbienne du sol). Par conséquent, la pérennité d'un tel système est plus fragile chez le bouleau que chez le châtaignier.

Nous voudrions tempérer ces conclusions par le fait que nous n'avons observé que des souches de bouleau n'ayant subi qu'une seule rotation. Même dans ce cas, il a été observé un renouvellement de racines (souche $\mathrm{n}^{\circ} 3$ ).

La question qui se pose alors est de savoir si l'induction du renouvellement des racines permet une plus grande pérennité du système racinaire, voire un accroissement de la productivité des parties aériennes.

\section{Conclusion}

Les résultats de cette étude ont été obtenus à partir de l'examen de trois souches de bouleau et de sept souches de châtaignier. Il convient donc d'être prudent et de vérifier ces résultats par des mesures complémentaires.

Il apparaît que les racines de bouleau, traité en taillis, observées après une rotation, ont un âge égal à celui de la souche. 
En revanche, les racines de châtaignier, quelque soit le nombre de rotations, ont un âge inférieur à celui de la souche.

Au cours du présent travail ont été mises au point différentes techniques (extraction, examen de cernes, datations) permettant l'étude de souches d'arbres traités en taillis.

Ces enseignements vont nous permettre de poursuivre ces recherches sur un nombre plus important de systèmes racinaires, et d'étudier d'autres espèces forestières.

\section{Remerciements}

Cette étude n’aurait pu avoir lieu sans le concours de :

- MM. Bonnaire et Soulier (Office National des Forêts).

- MM. Bendali, Cordon, Moreau, Romary (I.N.R.A., Forêt-Environnement).

\section{Summary \\ Study of root rings in coppiced trees}

Ten stumps of birch and sweet chesnut, managed under coppice system, were extracted. Sampling of roots were taken at the proximal part of stump.

Rings of these samples were examined. It appears that radial root growth is yearly.

In first approach - other measures are needed - the roots of birch are used by new sprouts. On the contrary the sweet chesnut seems to develop and use new roots.

Reçu le 17 décembre 1982.

Accepté le 14 avril 1983.

\section{Références bibliographiques}

Auclair D., Metayer S., 1980. Méthodologie de l'évaluation de la biomasse aérienne sur pied et de la production en biomasse des taillis. Acta Oecol., Oecol. Applic., 1 (4), 357-377.

Bedeneau M., Rimsky A., 1976. Radiographies de carottes de bois en rayonnement monochromatique. Ann. Sci. For., 33 (1), 19-26.

Вонм W., 1979. Methods of studying root systems. Springer Verlag, Berlin - Heidelberg - New York.

FAYLE D.C.F., 1968. Radial growth in tree roots. Distribution, timing, anatomy. University of Toronto, Faculty of Forestry. Technical Report n" 9, 168 pp.

FritTs H.C., 1976. Tree rings and climate. Academic Press, London-New York - San Francisco, $567 \mathrm{pp}$.

Laitakari E., 1935. The root system of Birch. Acta Forestalia Fennica, 41, 169-216.

LEES J.C., 1981. Three generations of red maple stump sprouts. Maritime Forests Research Center. Information report M-X-119, pp. 1-9.

Pages L., 1982. Etude méthodologique de l'effet du recépage sur le système racinaire de bouleau. E.N.I.T.E.F., rapport de $3^{\circ}$ année, 105 pp.

Pages L., Bedeneau M., Evin J., Pachiaudi C., 1983. Détermination de l'áge de cernes racinaires de taillis' de bouleau à l'aide du radiocarbone naturel d'origine thermonucléaire. C.R. Acad. Sci., Paris, III, 34.

Riedacker A., 1976. Rythmes de croissance et de régénération des racines des végétaux ligneux. Ann. Sci. For., 33 (3), 109-138.

Stout B.B., 1956. Studies of the root system of deciduous trees. Black Rock For. Bull., 15, $1-45$. 